Eurostudia

\title{
Une dévotion ordinaire
}

\section{Alexandre Legault}

Volume 11, numéro 1, 2016

\section{Feuilleton}

URI : https://id.erudit.org/iderudit/1036319ar

DOI : https://doi.org/10.7202/1036319ar

Aller au sommaire du numéro

\section{Éditeur(s)}

Le Centre canadien d'études allemandes et européennes

ISSN

1718-8946 (numérique)

Découvrir la revue

Citer cet article

Legault, A. (2016). Une dévotion ordinaire. Eurostudia, 11(1), 63-70.

https://doi.org/10.7202/1036319ar

Tous droits réservés ( Le Centre canadien d'études allemandes et européennes, 2016
Ce document est protégé par la loi sur le droit d'auteur. L'utilisation des services d'Érudit (y compris la reproduction) est assujettie à sa politique d'utilisation que vous pouvez consulter en ligne.

https://apropos.erudit.org/fr/usagers/politique-dutilisation/
Cet article est diffusé et préservé par Érudit.

Érudit est un consortium interuniversitaire sans but lucratif composé de l’Université de Montréal, l'Université Laval et l'Université du Québec à Montréal. Il a pour mission la promotion et la valorisation de la recherche. https://www.erudit.org/fr/ 


\section{Alexandre Legault \\ Université de Montréal}

Porté par un nouvel intérêt sur la religion et la matérialité, je suis retourné rendre visite à Cécile, une femme de 76 ans dont j'avais déjà tracé le parcours de vie à l'hiver de 2015 pour une petite enquête sociologique sur le vieillissement. De manière inattendue, j'avais été interpelé par son attitude ambivalente envers la religion, et six mois plus tard, je comptais saisir cette tension à travers l'observation et la prise de photographie, à son domicile, d'objets de la transcendance. En effet, je me suis questionné plus généralement sur le rapport qu'entretenaient les gens avec les artéfacts susceptibles de les accompagner dans les transcendances quotidiennes.

À l'instar des premières rencontres, Chanel, une amie de longue date, m'accompagnait voir Cécile. Comme elle est sa petite-fille, les entretiens sur fond de retrouvailles familiales ont apporté une dose opportune de spontanéité et de franc-parler, allégeant ainsi les interactions.

«En général, je m'intéresse aux objets ayant une signification particulière, une valeur sentimentale, ou bien rattachés à un souvenir », ai-je expliqué à Cécile. Je lui ai d'abord demandé si elle détenait des objets de ce genre qui, par exemple, lui rappelleraient sa mère. Elle m'a envoyé chercher sur sa table de chevet un cadre qu'elle avait commencé à exposer depuis trois ou quatre mois. Sur le portrait en buste, la mère s'y révélait jeune et élégante, un collier de perles au cou, l'air aimable. Cécile n'était pas adepte des vieilles photographies : "J'aime pas ça les photos. J'trouve ça plate moi regarder les gens que ça fait 40 ans qui sont décédés, au temps qui étaient jeunes... Tu changes, là !». Elle préférait, disait-elle, se souvenir de ses proches par la simple « pensée »... 
Par un heureux imprévu, Benoit, le neveu de Cécile d'une cinquantaine d'années, était en notre compagnie ce jour-là. Sans le lui avoir demandé, celui-ci m'a confié qu'il affichait un nombre impressionnant de portraits d'ancêtres sur les murs de sa maison. J'ai appris que lorsque Benoit traverse la cuisine, il prend le temps de saluer ses parents : "J'regarde la photo, pis j'parle à ma mère ». Il a aussi une boite dans sa chambre dans laquelle il a rassemblé les cendres de son père et de sa mère : "Moi, j'les ai mis ensemble. » Ainsi, avant de se mettre au lit, Benoit leur souhaite une bonne nuit, et au réveil, une bonne journée!

"Vous autres », a répondu Cécile à Benoit, "vous êtes très croyants. Moi, j'crois à rien. » Mais Cécile, qui ne croyait à rien, rassemblait aussi des objets se rapportant à de proches défunts. Ainsi, dans sa chambre, le portrait de sa mère était réuni auprès d'une lampe de chevet qu'avait possédée son père. En face du lit, une étagère arborait les photographies de ses deux sœurs, aujourd'hui décédées. Juste au-dessus était assise une poupée de porcelaine vieille d'une centaine d'années : elle avait appartenu à l'arrière-grand-mère de Chanel.

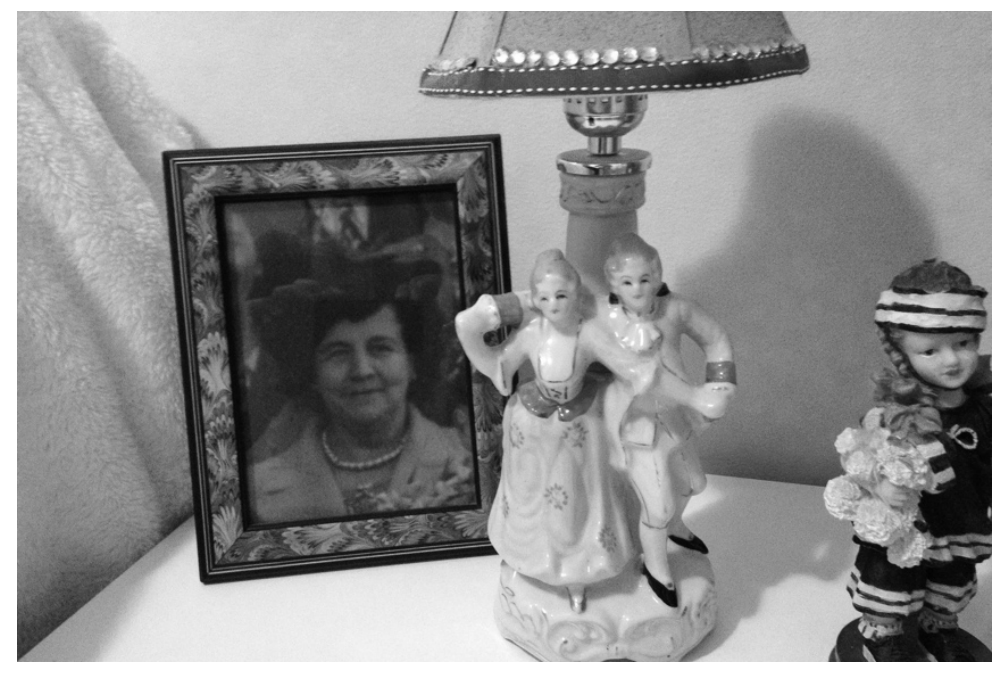

Photo 1. Sur la table de chevet de Cécile, une photographie de sa mère et une lampe ayant appartenu à son père.

Cécile et son mari Claude avaient une quarantaine d'albums photo, qu'ils avaient soigneusement rangés dans l'armoire de la bibliothèque du salon. De ce que j'ai pu en observer, la valeur que les gens accordent à ces clichés par milliers est souvent ambiguë. En raison de leur grand nombre, ils nous apparaissent en 
tant que pure trivialité. "J'ai des photos partout», répétait Cécile. "J'ai des diapositives, j'ai des films, j'ai toutes sortes de choses. » L'emploi du mot «tragique » serait démesuré pour qualifier une situation dans laquelle seraient perdus à jamais ces souvenirs sous forme documentés. Malgré tout, et ne serait-ce que pour y avoir pensé, un malheureux subirait la réprobation de ses proches s'il se départait volontairement d'un vieil album de famille.

Au souvenir de Cécile, l'album de mariage avait été le seul bien épargné des flammes lors d'un incendie ayant ravagé leur appartement en 1967. Bien que la fumée ait jauni les coins des pages, les photos, d'une qualité remarquable pour l'époque, étaient restées dans un état quasi impeccable. Il s'agissait bien de l'œuvre d'un professionnel. Toutefois, ce dernier, de manière plus efficace que l'aurait fait un amateur, reproduisait les stéréotypes les plus ordinaires dans des mises en scène idéalisées. Cécile ne semblait pas émue par toutes ces poses hollywoodiennes qu'elle avait un jour incarnées. En fait, le quotidien de l'époque apparaissait bien plus fidèlement dans les petits détails, qui, renvoyés à l'arrièreplan, auraient été oubliés par un peintre épris d'idéalisme. Par exemple, derrière une pose où l'on voyait Cécile en robe de mariée, solennellement agenouillée auprès de son père, se cachaient dans l'ombre les cadeaux entassés : un grillepain, une marmite, une planche à repasser et des lampes à abat-jours. De ce qui semblait a priori sans importance rejaillissaient les souvenirs : "Mon frère m'avait acheté un set de lampes, pis il les avait pas payées... Faique ils sont venus chez nous pour les saisir. J'ai dit: "laisse-moi-les, il m'a fait un cadeau”, mais il était plus capable de payer. "Bin là, mets-le sur mon compte, j'vais les payer !" »

Dans un album photo réservé aux six petits-enfants de Cécile et de Claude étaient regroupés des évènements que l'on avait jugé dignes d'être immortalisés : les anniversaires, les photographies scolaires, la fête d'Halloween et le Noël des campeurs, et puis les baptêmes. Malgré l'absence de «croyants » dans la famille, tous les petits-enfants avaient été baptisés. Lorsqu'était venu le temps de fêter cette occasion pour Chanel, un gâteau avait été préparé par Cécile et sa nièce. Sur la photo du dessert, on pouvait lire un court poème en acrostiche : 
Chanel notre amour pour toi est éternel

Honneur tu es pour papa et maman

Aujourd'hui c'est une journée très spéciale

Ne voulant que ton bonheur

Et l'amour tout au long de ta vie ; on te baptise

Le plus beau cadeau que Dieu nous a donné,

C'est toi Chanel!

Chanel a senti le besoin de justifier l'élan de ferveur de sa grand-mère : «Elle s'est dit qu'il fallait parler de Dieu, quand même... »

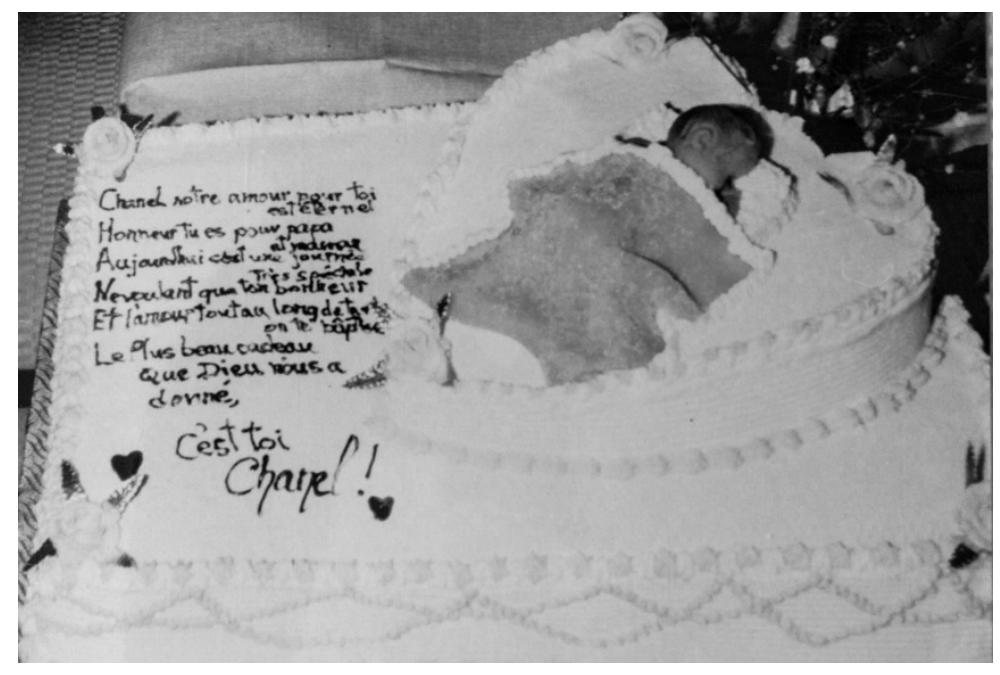

Photo 2. Une photographie d'un gâteau pour célébrer le baptême de Chanel.

À plusieurs moments, Cécile s'est montrée réticente envers les photographies d'ancêtres qu'on expose au mur, une pratique qui, selon elle, était très répandue dans sa famille. "Parce que les photos, j'aime pas ça », m'a fait part Cécile. "J'mets pas ça sur les murs. J'aime vraiment pas ça. J'ai juste des portraits de Chanel que j'mets sur le frigidaire. " La discussion sur ces photographies ouvrant rapidement sur les croyances et les superstitions, Cécile n'hésitait pas à manifester son agacement. Certes, dans le salon prévalaient les portraits des deux parents et du grand-père de Claude, mais justement, elle et son mari voulaient «s'en débarrasser »! 
Comme pour contrarier Cécile, ces objets étaient disposés dans l'espace de manière à optimiser leur visibilité. À l'avant-plan, deux portraits picturaux de formes ovales, inspirés du réalisme photographique, représentaient les parents de Claude. Bordés d'un cadre doré, ils avaient le teint pâle, la chevelure soignée, et étaient accoutrés de leurs plus beaux habits. À n'en juger que par cette mise en scène, Claude serait issu de la bourgeoisie... Puis, en direction de la sortie du salon, on pouvait voir sur un grand imprimé le grand-père de Claude dans son atelier, en train de peindre le portrait d'une femme. Voici donc où se cachait l'artiste derrière les deux portraits précédents. En dépit de ces manigances, j'ai voulu comprendre pourquoi, manifestement contre la volonté de Cécile, ces cadres avaient réussi à s'imposer sur les murs. Elle a répondu : "On les met là, c'est parce que ceux qui nous les ont donnés, ils voulaient les mettre sur le mur, et ils sont morts aujourd'hui. »

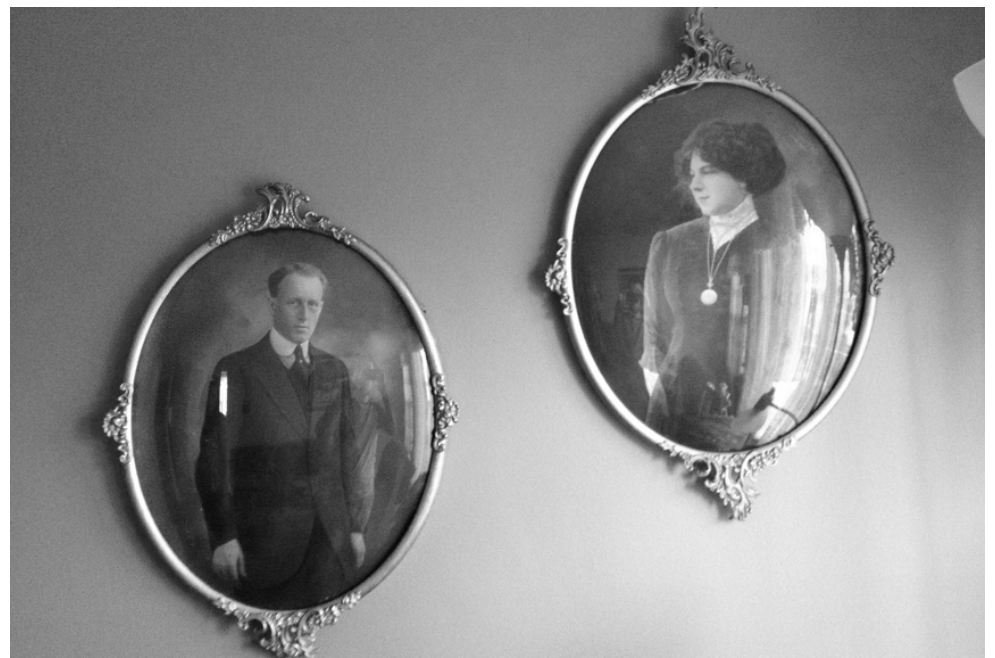

Figure 3. Accrochés au mur ouvrant sur le salon, les portraits des parents de Claude.

Dans l'étroit vestibule menant à la cour extérieure, on pouvait apercevoir au mur une vieille photographie en couleurs délavée par le temps, représentant un jeune joueur de hockey. La photographie n'était signée par nul autre que Claude. Puis, en face, comme pour remplacer un crucifix, se trouvait au-dessus de la porte du garde-robe une photographie laminée. On y reconnaissait René Lévesque - un acteur politique important pour le mouvement souverainiste au Québec - en compagnie du frère de Claude. Curieux, j'ai demandé pourquoi cette 
photographie s'était retrouvée à cet endroit précis. «Bin! Parce que c'tait René Lévesque! », m’a répondu Claude.

Une amie me racontait comment sa grand-mère croyait qu'à sa mort, elle rejoindrait son mari «au ciel», alors qu'elle pensait aussi qu'il n'y avait rien après la vie. De telles hésitations ne se sont jamais manifestées chez Cécile, du moins en ma présence. Même après avoir frôlé la mort dans un état de coma, il y avait de cela quelques semaines, elle n'avait pas fait de concession : "J'te le dis que si c'est ça mourir, c'est rien. Parce que t'as connaissance de rien. » De même, elle ne pouvait, contrairement aux autres membres de sa famille, consoler sa sœur mourante en lui assurant qu'elle irait rejoindre ses proches « au ciel»: «J'ai jamais été capable de dire ça. Parce que j'pas croyante, premièrement. Quand tu meurs, tu meurs. »

Prenant ces paroles au sérieux, j'ai été tout à fait étonné de croiser sur mon passage tous ces objets évoquant la religion, la spiritualité ou l'ésotérisme. Ainsi, m'arrêtant sur une mystérieuse roue en pierre arborant les douze signes zodiaques, j'ai demandé à Cécile ce qu'elle pensait de l'astrologie. Sans équivoque elle m'a expliqué qu'il s'agissait là d'une vulgaire supercherie! Et la croix chrétienne au mur de la chambre à coucher? Et bien, celle-ci n'aurait aucune signification religieuse. À leur anniversaire de mariage, elle avait été donnée en cadeau par l'ex-conjointe de son fils et symboliserait l'union. Voici donc pourquoi, à l'intersection des segments de la croix, deux anneaux s'entrelaçaient, ce que Claude n'avait jamais remarqué jusqu'à ce jour. Mais alors, comment comprendre cette statuette en céramique, posée au mur adjacent, représentant Jésus-Christ? Et que dire des nombreuses figurines d'anges et de saints qui peuplaient les étagères, les têtes de lits, ou bien qui étaient suspendues à la chaine du ventilateur au plafond ? 


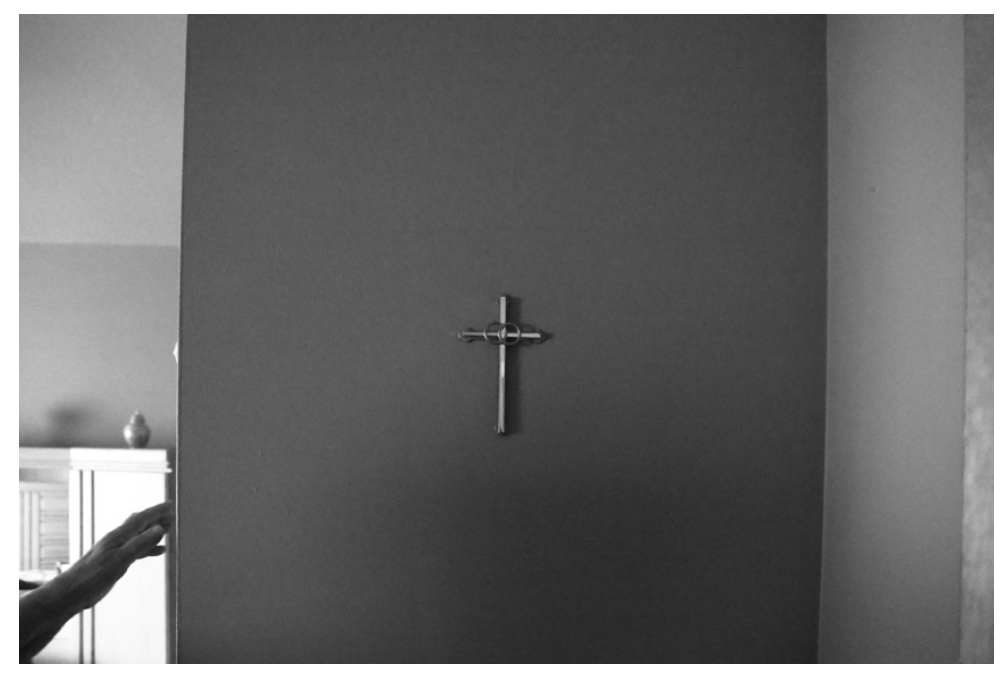

Photo 4. Une croix accrochée au mur de la chambre à coucher.

Tout au long de l'enquête, je voulais connaitre la signification cachée de tous ces étranges objets ; c'était sans succès. Quoi qu'il advienne, quelle sorte de vérité aurais-je obtenue? Dans le cas de Cécile, j'ai dû me contenter provisoirement des réponses qui m’ont été données. Ces choses, plus ou moins anciennes, avaient été transmises ou données par des proches, parfois achetées, et à l'évidence, elles avaient été accumulées avec le temps. Elles formaient des traces matérielles du réseau de relations sociales dans lequel Claude et Cécile étaient inscrits.

La raison pour laquelle le cas de Cécile m'interpelait au sujet de la religion matérielle ne m'est apparue plus clairement que dans les semaines subséquentes à la rencontre. Ne s'identifiant à aucune communauté religieuse, ses attitudes à l'égard des objets qui furent ici exposés permettaient de faire voir, en amplifiant ses traits, un aspect de la vie qui nous concerne tous et à laquelle on porte peu attention au quotidien.

Comme Cécile, nous possédons à la maison de ces choses qui, si elles ne sont ni désirées ni détestées, traînent dans le garde-robe, s'affichent sur les murs ou sont posées sur nos tables et nos commodes. Ce sont des objets reçus en cadeau, légués par un vieil oncle, ou bien rapportés de voyage. Ce sont de vieux livres qu'on n'ouvrira plus, des albums photo qu'on ne regarde jamais. Pourquoi 
nous en encombrons-nous ? Ces choses, sans être chargées d'un sens profond ou d'un effet magique, partagent toutefois un sens sacré: un caractère sacré ordinaire qui se révèle dans l'interdit de jeter ou de vendre. À défaut d'outrepasser cet interdit, elles sont appelées à s'accumuler jusqu'à ce que la vie nous force à nous en séparer ou qu'arrive l'occasion de les transmettre à un héritier.

Qu'adviendra-t-il de la poupée de céramique, des bibelots, des portraits d'ancêtres, de la vieille lampe de chevet, des quarante albums photo ? La plupart de ces artéfacts ne correspondent malheureusement plus aux goûts du jour! Faute de preneur, Cécile s'attend à ce qu'à son décès tout cela se retrouve aux ordures. Mais elle ne s'en inquiète pas. Les contraintes du sacré ordinaire opèrent dans les limites de la vie quotidienne, et, une fois traversées, il faut bien savoir lâcher prise. 